\title{
On Developing SeamlessSpectrum Handoff Strategyfor Cognitive Radio Networks
}

\author{
A. I. Abu Tair ${ }^{1}$, M.-T. El Astal ${ }^{2}$, M. Shaat ${ }^{3}$, F. El-Nahal ${ }^{4}$ \\ ${ }^{1}$ CTRC, P-ICTRA, Palestine, aabutair@p-ictra.org \\ ${ }^{2}$ CTRC,P-ICTRA, Palestine, mtastal@p-ictra.org \\ ${ }^{3}$ Senior Researcher, CTTC, Spain, musbah.shaat@cttc.es \\ ${ }^{4}$ Electrical Engineering IUG, Palestine, fnahal@ingaza.edu.ps
}

\begin{abstract}
In Cognitive Radio (CR) systems, the spectrum handoff is a key-factor as it could affect propagation path nature. Accordingly, it may affect the overall system coverage and performance. In this article, a new spectrum-handoff strategy is proposed in order to minimize the occurrence of spectrum handoff and hence to achieve a better transmission performance. Specifically, it provide a better channel-selection strategy combined with a power adaptation scheme. This is to reduce/avoid interference and also to conserve power consumption in the system and hence get a greener communication system. The simulation results show an enhancement of up to $64 \%$ in switching requests. Also, the system is analyzed in terms of BER and compared to others to show the performance maintained.
\end{abstract}

Index Terms — spectrum handoff, cognitive radio.

\section{INTRODUCTION}

Cognitive Radio (CR) technology is one of the most evolving communications technologies. This is because it has a greater potential to utilize efficiently the limited RF spectrum. The basic idea is that the CR terminals (termed as secondary user (SU)) can use a temporal-vacant channel and vacate this channel immediately once the licensed-primary users (termed as primary user (PU)) get back. The SU has to make sure that it avoids any harmful interference with the licensed channels. While vacating the SU needs to suspend its transmission and handoff to another vacant channel; this kind of handoff is termed as spectrum handoff [1]. Spectrum handoff is a very challenging issue in CR networks. This is because it difficult to accomplish a seamless transition in very short period while maintaining better performance and avoid harmful interference to others.

In literature, a number of algorithms were proposed to reduce/handle the number of spectrum handoff in $\mathrm{CR}$ networks $[2,3,4]$. A novel spectrum handoff scheme was proposed considering an adjustable power control in order to avoid some of the handoffs [5]. In [6], the effect of the different path loss of different frequencies was studied and also an algorithm was proposed in order to overcome these effects limitations on the spectrum handoff. The quality of service (QoS) can degrade significantly due to a change of frequency which reduced the outage probability. The spectrum access scheme of unlicensed channels as backup improves and characterizes the spectrum handoff performance: link maintenance probability, the number of spectrum handoff, and switching delay $[7,8]$. The spectrum decision algorithm based on prediction is proposed to decrease spectrum handoff probability which can achieve fewer disruptions to primary transmissions by letting SUs proactively predict the future spectrum availability and perform spectrum handoffs before a PU occupies the current spectrum[9,10,11]. Wang et al. in [12] and Zhang et al. in [13] use queuing network model to discuss the effect of proactive sensing and reactive sensing on spectrum handoff to characterize the spectrum usage behaviors between primary and secondary users in CR networks dependent of sensing time. Voluntary spectrum handoff is used to reduce temporary communication disruption time [14]. Some other research consider a particular communication networks like as Adhoc and cooperative schemes in [15,16]

Here, a seamless spectrum-handoff strategy is proposed. It is based on a fixed sensing window, a variable history window and the reduced forced spectrum handoffs. Using this approach, SUs can have longer undisrupted connection. It depends on a systematic frequency selection scheme combined with adaptation power of transmitter base station. This is to achieve optimal usage of spectrum and resource. Also, improvement the overall performance $\mathrm{CR}$ system, reducing spectrum handoff from failure and decreasing the interference and power dissipated will be presented.

This paper is organized as follows. In Section II, the problem is illustrated and modeled. In Section III, a new handoff strategy is proposed. Section IV is includedMonte Carlo simulation results for different real network scenarios.

\section{Problem Illustration}

The main effect of spectrum handoff can be illustrated by examining the path-loss changing due to frequencies variation. Without loss of generality, it has been assumed that OkumuraHata (urban) path loss model is used [6]. It is given by

$$
L_{d B}=\alpha+\beta \log _{10} d-\gamma
$$

where

$$
\begin{aligned}
& \alpha=69.55+26.16 \log _{10} f_{c}-13.82 \log _{10} h_{b} 1 \mathrm{a} \\
& \beta=44.9-6.55 \log _{10} h_{b} 1 \mathrm{~b} \\
& \gamma=3.2\left(\log _{10}\left(11.7554 h_{r}\right)\right)^{2}-4.97_{1 \mathrm{c}}
\end{aligned}
$$

where $f_{\mathrm{c}}, d, h_{\mathrm{b}}, h_{\mathrm{r}}$ is the center frequency of channel used, the 
distance of link, the height of base station, and the height of receiver station, respectively.

By examining the factor $(\alpha)$, you can notice the effect results due to the spectrum handoff.The increasing in $f_{\mathrm{c}}$ value results in increasing in $\alpha$ value and hence higher path loss is experiencing. This would be a great challenge particularly as the CR networks have a wide spectrum [9]. For example, at distance of 30 kilometers, the path loss increases from 156.5 $\mathrm{dB}$ at $100 \mathrm{MHz}$ to $180.85 \mathrm{~dB}$ at $1700 \mathrm{MHz}$. This loss of 24.3 $\mathrm{dB}$ could significantly impact the link performance.

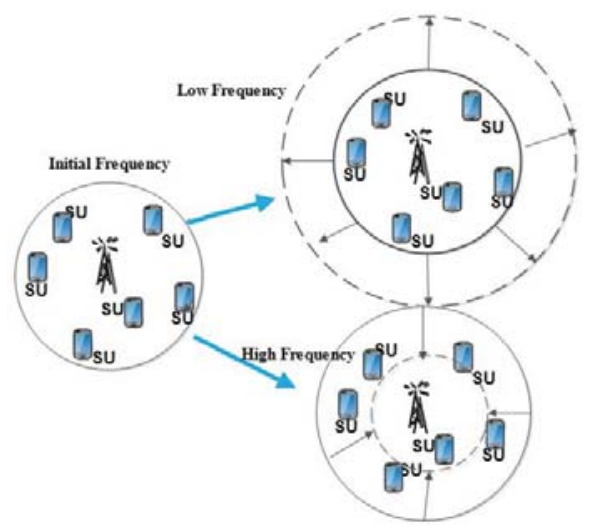

Figure 1: Impact of spectrum handoff on cell Coverage.

\section{Proposed Spectrum Handoff Strategy}

The strategy proposed is executed in two stages, illustrated graphically also in Figure $\mathbf{1 0}$ (at the end of article):

1.The first stage is for channel frequency selection process, where consists of two schemes. Namely:

- the systematic selection (proposed) where the search selection channel begins from the first channel in dedicated pool and moves to nearest neighbor channel until obtaining a free one to be assigned as channel handoff as shown in figure $3[17]$.

- The other option is to keep the selection process a random process, shown in figure 2 .

2.In the second stage which dedicated to the use poweradaptation enhancement [18]. This is to apply that on the result from selection scheme (may be high or low):

- With "power adaptation", the check is to examine the new power compared to the existing one. The algorithm calculates the new transmitted power based on new frequency selection using the famous formula of power transmission which is the transmit power equal the receive power (power sensitivity) subtract path loss value. Then, estimate this value to coverage all SUs in the cell area (high to low case); otherwise use the maximum power of the system to estimate the cell coverage (low to high). From this approach, the reserved power is achieved and the number of handoff is decreased as shown in the simulation results in next section.

- With "no power adaptation", the check is about new frequency selection only, since the coverage area is a reduced or expanded according to new frequency selection (spectrum handoff).


Figure 2: Spectrum Band random Selection (classic) a) Initial state; b) Low to High HO; c) High to Low HO; d) Drop HO; e) Block HO.

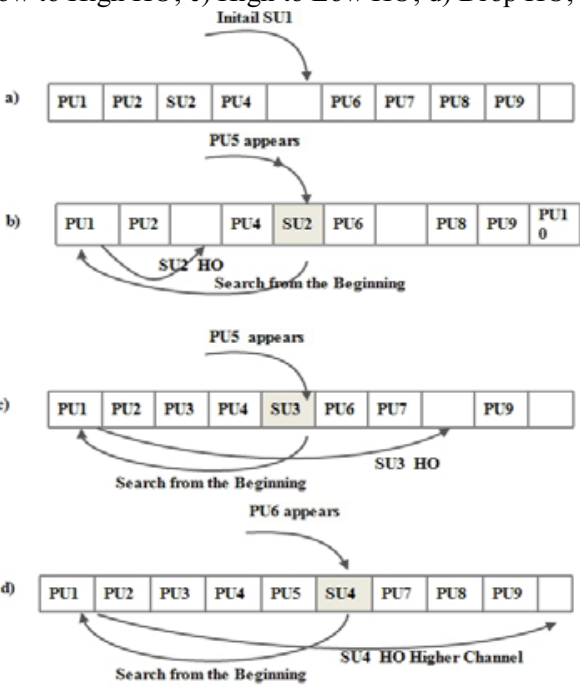

Figure 3: Spectrum Band Systematic Selection a) Initial state; b) Low to High; c) High to Low; d) Highest HO.

\section{Performance And Simulation Results}

This section investigates the performance of the strategy proposed over 3 different real scenarios to express the situations of small, medium, and large communications cells [19]. It should be mentioned that four variants of selection schemes are analyzed; the random selection without power adaptation (existing), random selection with power adaptation (modified existing scheme), and systematic selection without power (proposed), and systematic selection with power adaptation (proposed).

\section{- Case I: Small Cell Application to CR}

A case of either Femtocell or Wi-Fi [20] is considered. This expresses the situation of small cell scenarios. The simulation parameters are given in Table 1. 


\begin{tabular}{|l|c|c|}
\multicolumn{1}{|c}{ Parameters } & Femtocell & Wi-Fi \\
\hline Frequency Range MHz & From 54 to $700 \mathrm{MHz}$ \\
\hline Tx Power $(\mathrm{dBm})$ & $10-20$ & $20-23$ \\
\hline Height $\mathrm{h}_{\mathrm{b}}(\mathrm{m})$ & $3-6$ & $3-12$ \\
\hline Cell Size Coverage $(\mathrm{m})$ & $20-50$ & $100-200$ \\
\hline number of User in Cell & $3-10$ & $10-30$ \\
\hline Rx Sensitivity $(\mathrm{dBm})$ & -62 & -62 \\
\hline Channel Model & \multicolumn{2}{|c|}{ Okumora-Hata } \\
\hline
\end{tabular}

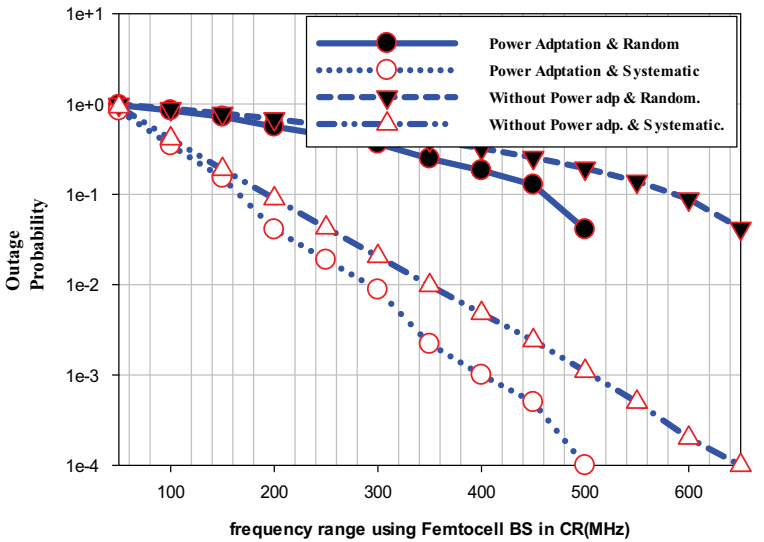

Figure 4: Outage probability of services shown in Case I (FemtoCell)

From Fig. 4, you can conclude that in a case of " random selection scheme without adaptation " a BS-frequency range lower than $580 \mathrm{MHz}$ is not efficient to get outage probability of 0.1. This is because, all the switching frequencies are lower than the reference probability. In the other words, the outage probability increases if we use any frequency BS lower than this value which means that most the switching frequency transit from high to low. In contrast, this is decreases to up to 400 using "random selection with power adaptation". Using the proposed methods, this decreased to a frequency range of nearly $180 \mathrm{MHz}$ to achieve the same outage probability reference. From these result, you can conclude that the proposed scheme is more efficient and the overall system performance is enhanced. Namely, the frequency range is reduced by $420 \mathrm{MHz}$. In other words, the outage probability is enhanced from $>0.1$ by the existing/ modified existing selection schemes to $0.0001-0.00001$ outage probability.

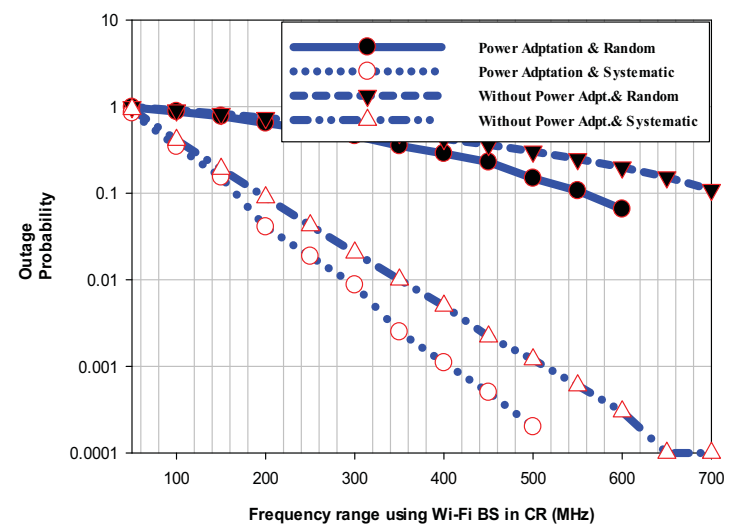

Figure 5: Outage probability of services shown in Case II (WiFi).
Fig. 5 consider the case of using Wi-Fi BS in frequency range of TV WS. It is clear that the proposed systematic selection scheme also improves (decrease) the frequency range by up to $520 \mathrm{MHz}$ compared to reference scheme.From result of Fig. 4 and 5, you can expect that there is an improvement by $70 \%$ in the switching range availability from all range of frequency band in case of small-cell applications.

\section{- Case II: Medium Cell Application to CR}

Here, both the WiMAX [21] and LTE 700 [22] as real example applications of medium cell size are considered.
Parameters
Fixed WiMAX
LTE 700

Available Frequency $\quad$ From 54 to $700 \mathrm{MHz}$

\begin{tabular}{l|l|l} 
Tx Power $(\mathrm{dBm})$ & $36-40$ & $40-42$ \\
\hline
\end{tabular}

Height $\mathrm{h}_{\mathrm{b}}(\mathrm{m}) \quad 18-30 \quad 18-30$

\begin{tabular}{l|l|l} 
Cell Coverage $(\mathrm{km})$ & Up to 15 & Up to 15
\end{tabular}

\# of User in Cell $\quad 50-150 \quad 50-150$

\begin{tabular}{l|l|l} 
Rx. sensitivity $(\mathrm{dBm})$ & -107 & -107
\end{tabular}

\section{Channel Model Okumora -Hata}

Table 2 System parameters in medium cell size

Similar to Case I, the system considered are evaluated using the four-variant of selection schemes.It is clear that the proposed selection schemes decreases the frequency range for WiMAX and LTE up to $450 \mathrm{MHz}, 460 \mathrm{MHz}$ respectively compared to the classic random scheme as shown in figure 6,7 . This also can be expressed in term of outage probability, the outage probability is enhanced 0.0001-0.00001 instead of $>0.1$. Comparing the gain of wide frequency for two applications WiMAX and LTE in this case and measured in all range of frequency, it is more efficient and get availability up to $64 \%$ from all range of frequency band.

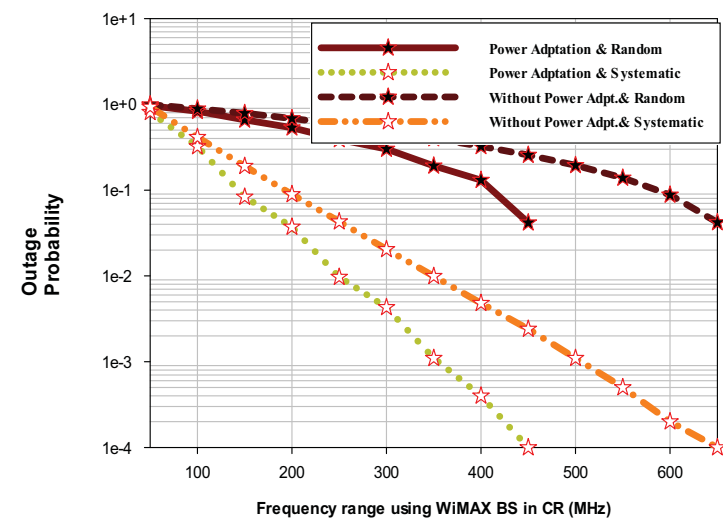

Figure 6: Outage probability of WiMAX CR application

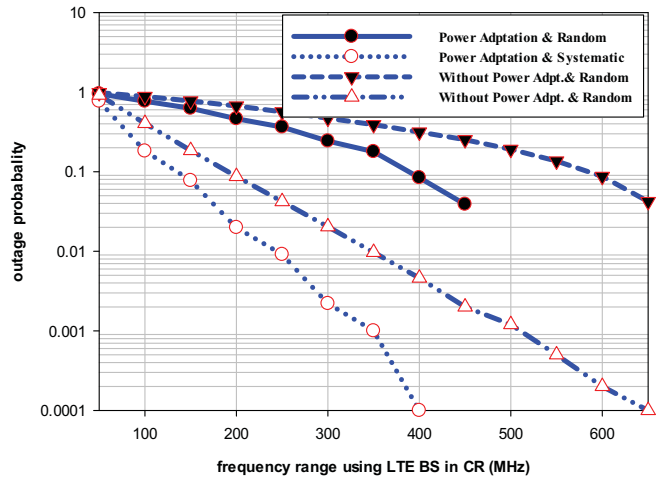

Figure 7: Outage probability of services shown in Case II (LTE). 
- Case III: Large Cell Application to CR

We considered characteristics of Public Safety [23] and IEEE 802.22 [19] as example applications from real environment in large cell size. The simulation parameters are given in following Table 3 .

Table 3 System parameters in large cell size

\begin{tabular}{|c|c|c|}
\hline \multicolumn{1}{|c|}{ Parameters } & Public safety & IEEE802.22 \\
\hline Available Frequency & From 54 to $700 \mathrm{MHz}$ (TV WS) \\
\hline Tx Power (dBm) & $42-45$ & $40-46$ \\
\hline Height hb (m) & $18-30$ & $18-30$ \\
\hline Cell Size (km) & $10-30$ & $20-40$ \\
\hline of User in Cell & $50-150$ & $70-150$ \\
\hline Rx. sensitivity (dBm) & -119 & -114 \\
\hline Channel Model & \multicolumn{2}{|c}{ Okumora -Hata }
\end{tabular}

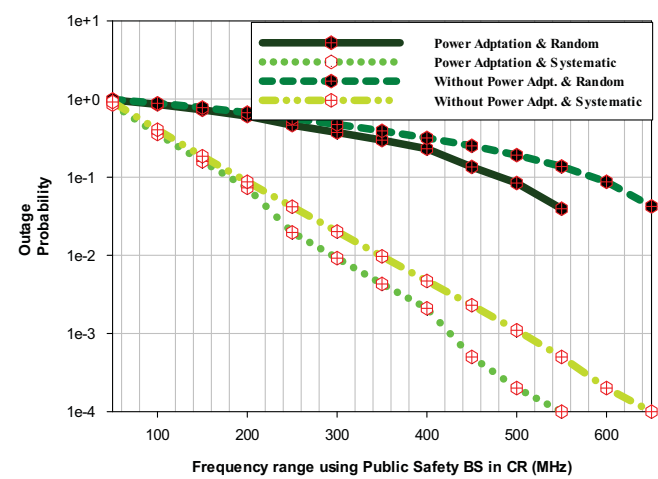

Figure 8: Outage probability of services shown in Case III (PS).

Figure 8, 9 shows the performance gain of the proposed scheme using two applications in large cell size public safety and IEEE 802.22 in frequency range to note the impact frequency selection in this case. Generally, the proposed systematic selection scheme improves the performance of wide frequency switching gain (spectrum handoff) for Public Safety and IEEE 802.22 up to $410 \mathrm{MHz}, 440 \mathrm{MHz}$ respectively comparing with classic random scheme.Comparing the gain of wide frequency from two applications Public Safety and IEEE 802.22 in this case referred to all range of frequency. We prove that the proposed systematic scheme is more efficient and improve the switching range availability up to $57 \%$ from all range of frequency band.

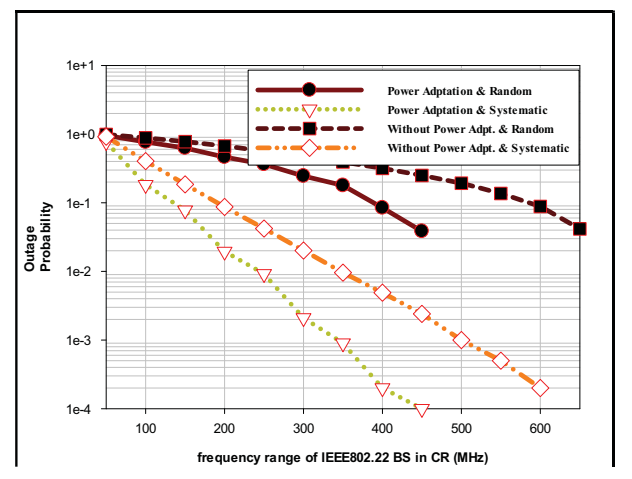

Figure 9:Outage probability of IEEE 802.22

\section{COMPARISON THE GAIN OF WIDE FREQUENCY SWITCHING RESULTS BETWEEN SCHEMES}

Table 4 shows the gain of a wide frequency transition (handoff) range in details. First, we display the impact of applying adaptation power in both classic random and proposed systematic schemes. We note that the effect is considered acceptable to expand the range of frequency switching (handoff), since it is reach up to $190 \mathrm{MHz}$ in classic scheme while $70 \mathrm{MHz}$ in proposed scheme in three cell coverage sizes (small, medium, large)cases. Second, we present the gain value of proposed scheme up to $520 \mathrm{MHz}$ which is considered as the worst classic scheme (No Adaptation power and random), also in three cell coverage size cases. Accordingly, the proposed scheme increases the wide of the frequency range by about $70 \%, 64 \%, 57 \%$ approximately in the three cases of cell coverage size .This ensures that the proposed scheme can be adopted in many applications and in different cell sizes and environments.

\section{CONCLUSION}

Spectrum handoff is one of the most important issues in CR networks. This paper proposed two seamless spectrum handoff schemes that is improved the performance of the CR networks. It reduce the number of spectrum handoff from falling, dropping, or breaking, so channel assignment in CR networks. The algorithms allows to choose the selection method between systematic or classic random. Moreover, the adaptation of power is also introduced to get better performance.

\section{REFERENCES}

[1] J. Wang, M. Ghosh, K. Challapali, "Emerging cognitive radio applications: A survey," Communications Magazine, IEEE, March 2011, vol.49, no.3, pp.74-81.

[2] J. Thomas and P. P. Menon, "A survey on spectrum handoff in cognitive radio networks," 2017 International Conference on Innovations in Information, Embedded and Communication Systems (ICIIECS), Coimbatore, 2017, pp.1-4. doi: 10.1109/ICIIECS.2017.8275896

[3] V. Ramani and S. K. Sharma, "Cognitive radios: A survey on spectrum sensing, security and spectrum handoff," in China Communications, vol. 14, no. 11, pp. 185-208, Nov.2017. doi: 10.1109/CC.2017.8233660

[4] M. E. Bayrakdar and A. Çalhan, "Comparative performance evaluation of efficient spectrum handoff methods in wireless cognitive networks," 2018 26th Signal Processing and Communications Applications Conference (SIU), Izmir, 2018, pp. 1-4. doi: 10.1109/SIU.2018.8404573

[5] L. Dianjie, X. Huang, C. Liu, J Fan, "Adaptive Power Control Based Spectrum Handover for Cognitive Radio Networks." In 2010 IEEE Wireless Communications and Networking Conference (WCNC), 2010, pp. 1-5.

[6] J. Ohyun, D. H. Cho,"Seamless spectrum handover considering differential path-loss in cognitive radio systems." IEEE Communications Letters, Mar.2009, no. 3, pp. 190-192. 
[7] K. A. Mohamed, H. Al-Mahdi, A. M. Thiel, "Spectrum handoff reduction for cognitive radio ad hoc networks." In 2010 7th International Symposium on Wireless Communication Systems (ISWCS), IEEE, pp. 1036-1040.

[8] Y. Zhang, "Spectrum Handoff in Cognitive Radio Networks: Opportunistic and Negotiated Situations," IEEE Int'l. Conf. Commun (ICC), June 2009.

[9] L. Yang, Y. Dong, H. Zhang, H. Zhao, H. Shi, X. Zhao, "QoS provisioning spectrum decision algorithm based on predictions in cognitive radio networks." In IEEE, 2010, 6th Inter. Con. On Wir. Comm. Net. and Mobile Comp.(WiCOM), pp. 1-4.

[10] S. Yi, J. Xie, "Prospect: A proactive spectrum handoff framework for cognitive radio ad hoc networks without common control channel." IEEE Transactions on Mobile Computing, Nov. 2012, no. 7, pp. 1127-1139.

[11]Y. Zhao, Z. Hong, Y. Luo, G. Wang and L. Pu, "Prediction-Based Spectrum Management in Cognitive Radio Networks," in IEEE Systems Journal. doi: 10.1109/JSYST.2017.2741448

[12]L. Ch. Wang, W. W. Chung, "Spectrum Handoff for Cognitive Radio Networks: Reactive-Sensing or Proactive-Sensing?", In EEE International Performance, Computing and Communications Conference IPCCC, 2008, pp. 343-348.

[13] X. X. Zhong, M. S. Hou, "Dynamic spectrum allocation scheme based on statistical information and multi-queue in CR networks," Journal of Jilin University (Information Science Edition), May 2009,pp.1-5.

[14] Y. S. UN, E. Ekici, "Voluntary spectrum handoff: a novel approach to spectrum management in CRNs." In 2010 IEEE Inter. Con. on Comm. (ICC), pp. 1-5.

[15] A. Shakeel, R. Hussain, A. Iqbal, I. Latif, S. A. Malik and B. Omoniwa, "Adaptive Spectrum Handoff Scheme in Cognitive Radio Ad-Hoc Networks," 2018 International Conference on Advances in Computing and Communication Engineering (ICACCE), Paris, France, 2018,pp.127-132. doi: 10.1109/ICACCE.2018.8441757

[16] W. Nasrin and J. Xie, "A Mobility Management Scheme to Reduce the Impact of Channel Heterogeneity in Cognitive Radio Femtocell Networks," 2016 13th Annual IEEE International Conference on Sensing, Communication, and Networking (SECON), London, 2016,pp.1-9. doi: 10.1109/SAHCN.2016.7732964

[17]T. A. Weiss, F. K. Jondral, "Spectrum Pooling: An Innovative Strategy for the Enhancement of Spectrum Efficiency," IEEE Comm. Magazine, Mar. 2004, vol. 42, no. 3, pp. 8-14.

[18] L. Dianjie, X. Huang, C. Liu, J Fan, "Adaptive Power Control Based Spectrum Handover for Cognitive Radio Networks." In 2010 IEEE Wireless Communications and Networking Conference (WCNC), 2010, pp. 1-5.

[19]C. Carlos, Ch. Kiran, B. Dag," IEEE 802.22: An Introduction to the First Wireless Standard based on Cognitive Radios", Journal of Comm., vol.1, no.1, APRIL 2006.

[20]H. LI, U. H, "Cognitive Femtocell Networks: An Opportunistic Spectrum Access for Future Indoor Wireless ", IEEE Wireless Communications, April 2013.

[21] http://transition.fcc.gov/pshs/techtopics/techtopics11.html
[22] ETSI, "LTE; Evolved Universal Terrestrial Radio Access (E-UTRA) Base Station (BS) radio transmission and reception" , 3GPP TS 36.104 versin 10.1.0 Release 10", Technical Specification, 2011.

[23] N. Jesuale, B. C. Eydt, "A Policy Proposal to Enable Cognitive Radio for Public Safety and Industry in the Land Mobile Radio Bands", proceedings of IEEE Inter. Symposium on New Frontiers in Dynamic Spectrum Access Net. (DYSPAN), April 2000 


\begin{tabular}{|c|c|c|c|c|c|c|c|}
\hline \multicolumn{2}{|c|}{ Parameters } & \multicolumn{2}{|c|}{ Small Cell Coverage } & \multicolumn{2}{|c|}{$\begin{array}{l}\text { Medium Cell } \\
\text { Coverage }\end{array}$} & \multicolumn{2}{|c|}{ Large Cell Coverage } \\
\hline & & Femtocell & Wi-Fi & WiMAX & LTE & Public & IEEE 820.22 \\
\hline \multirow{2}{*}{$\begin{array}{c}\text { Effect } \\
\text { power to } \\
\text { Gain wide } \\
\text { frequency } \\
\text { (MHz) }\end{array}$} & $\begin{array}{l}\text { Random } \\
\text { Classic }\end{array}$ & 120 & 130 & 180 & 190 & 103 & 190 \\
\hline & $\begin{array}{c}\text { Proposed } \\
\text { Systematic }\end{array}$ & 30 & 35 & 45 & 70 & 40 & 60 \\
\hline \multicolumn{2}{|c|}{$\begin{array}{l}\text { Gain wide frequency } \\
\text { between Proposed and } \\
\text { Classic scheme (MHz) }\end{array}$} & 400 & 520 & 450 & 460 & 410 & 440 \\
\hline
\end{tabular}

Table 4 Comparison the Gain of wide frequency switching Results between Schemes

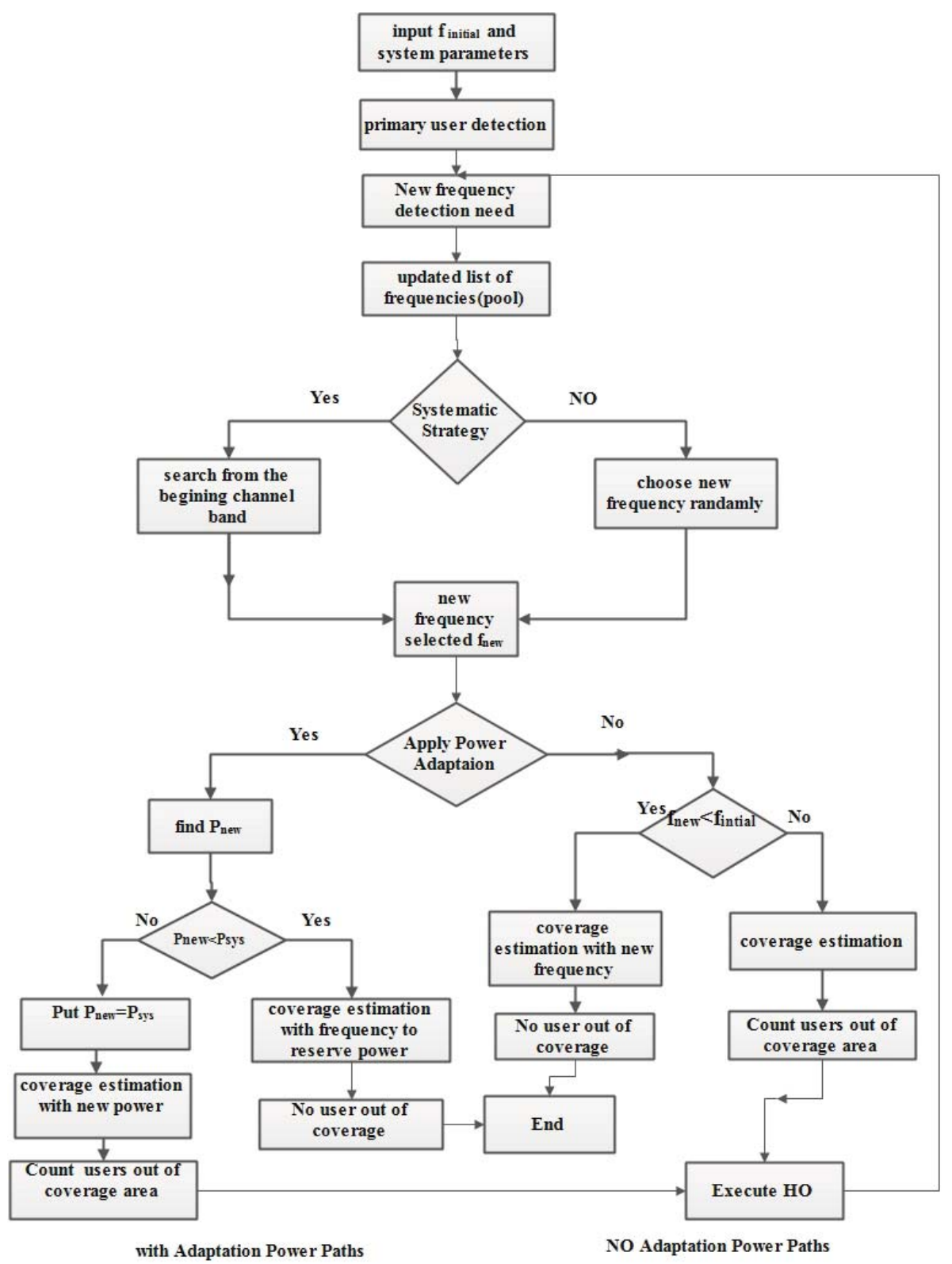

Figure 10: Flowchart Algorithm Proposed Scheme. 